\section{Uma revisão sobre instrumentos de rastreamento de violência doméstica contra o idoso}

\author{
A review of screening tools to assess \\ domestic violence against the elderly
}

Carlos Montes Paixão Jr. ${ }^{1}$

Michael E. Reichenheim ${ }^{2}$

\section{Introdução}

Data on elder abuse in Brazil are scarce. Systematic and thorough documentation can facilitate studies on magnitude, risk factors, and care of victims. This article reviews the existing screening tools on violence against elders in order to examine recent progress in the field, both in Brazil and abroad. A systematic review through MEDLINE, PsycINFO, LILACS, CANE, and OVID and reference books was performed using specific inclusion criteria. Studies on psychometric background and utilization of selected instruments were systematically examined, applying a set of quality assessment criteria. The Conflicts Tactics Scale was the only one of 17 identified scales that had been adapted to the Brazilian culture, although focusing on a younger age group. Some instruments with good psychometric characteristics were also found, namely the Caregiver Abuse Screen, Elder Abuse Assessment Tool, Hwalek Sengstok Abuse Screening Test, Indicators of Abuse Screen, QUALCARE scale, and Vulnerability to Abuse Screening Scale. However, there are still gaps in the process of design, validation, and adaptation, both in Brazil and abroad.

Domestic Violence; Aged; Psychometrics
A violência doméstica não é um fenômeno novo. Há vários indícios de que as violências social e doméstica estejam interconectadas 1 . Nos Estados Unidos, onde se tem estudado mais detalhadamente o evento, observa-se que as pessoas são mais freqüentemente mortas, agredidas fisicamente, feridas e lesionadas em seus domicílios por outro membro da família do que em qualquer outro ambiente ou por qualquer outro indivíduo 2 . Mesmo que envolva apenas alguns dos participantes do grupo, a violência doméstica tem repercussões em toda a família ou no ambiente da moradia ${ }^{3}$. Os custos envolvidos são também grandes, podendo ser monetários em decorrência do tratamento das conseqüências, da perda de dias de trabalho, dos custos legais e da diminuição de anos úteis por morte ou impedimentos graves; e não monetários, devido a dias de ausência escolar ou seqüelas psicológicas, dentre outras 3 .

Para que se conheça o fenômeno de forma mais completa, interessa estudar as várias facetas da violência que envolve indivíduos em relações de intimidade, quer por consangüinidade, quer devido a relações profissionais que se estabelecem no ambiente domiciliar. Uma das relações de crescente interesse concerne ao idoso, grupo etário no qual atos de negligência e violência doméstica passaram a aumentar em importância com a sua progressiva 
representatividade populacional desde o início do século passado 4 .

Ainda assim, foi somente nas duas últimas décadas que a questão começou a despertar real mérito na comunidade científica 5 . No âmbito do estudo da violência doméstica, aquela perpetrada contra os idosos foi a última a despertar o interesse da comunidade científica e a ser considerada uma questão de saúde pública. No Brasil, principalmente a partir da década de 1980, as mortes por acidentes e por violências de qualquer natureza vêm aumentando proporcionalmente como causa de óbitos, inclusive entre os idosos 6. Entretanto, importa recordar que a repercussão da violência que se abate contra o idoso não se restringe ao óbito. O fenômeno vem sendo vivenciado sob várias formas, muitas dessas não fatais. Expressa-se como atos violentos sofridos no seio da família, abandono em asilos, perda de direitos próprios ao exercício da cidadania, segregação por idade e, em última instância, ostracismo e exclusão social 7.

Estimativas sobre abuso em idosos são difíceis de obter-se devido ao caráter freqüentemente velado do problema. Essa subnotificação associa-se, muitas vezes, à solidão, ao isolamento e à tendência dos indivíduos idosos a não relatar suas adversidades 8 . Estudos norteamericanos e europeus indicam prevalências entre 3 e $4 \%$ 9,10,11. No melhor do conhecimento dos autores, não há, ainda, qualquer estimativa da prevalência do fenômeno no Brasil.

Várias organizações atuando diretamente com os idosos no âmbito clínico consideram que a identificação nos casos suspeitos seja também de responsabilidade do profissional clínico 12. Há poucas evidências sobre como, efetivamente, suspeitar ou reconhecer a presença de abuso, e são poucos os profissionais de saúde que ativamente rastreiam pacientes que não possuam alguma lesão aparente ${ }^{13}$. Todavia, há consenso que instrumentos apropriados de rastreamento de violência em idosos sejam cruciais para o progresso da prática e pesquisa desse importante problema de saúde 14 .

O presente estudo objetiva selecionar, na literatura de referência, os instrumentos mais profícuos para o rastreamento de violência contra o idoso. Especial foco é dado àqueles desenvolvidos para uso em ambiente clínico. Pretende-se avaliar os instrumentos quanto ao grau de utilização na literatura, bem como escrutinar os respectivos estágios de desenvolvimento, em especial, em relação ao estado da arte do conhecimento sobre as características psicométricas. A intenção é também identificar, dentre os selecionados, quais já foram adapta- dos para uso no Brasil e, na seqüência, apontar aqueles que, porventura, mereçam ser submetidos a um processo formal de adaptação transcultural.

\section{Métodos}

Na primeira etapa, o processo envolveu uma ampla revisão sobre as definições das diversas formas de violência em idosos. Essa revisão serviu, inicialmente, de base para a escolha de instrumentos que captassem violência doméstica, íntima ou familiar contra os mais velhos, e explicitamente excluir os que avaliavam outras formas de violência, tais como a institucional e social. Ademais, o detalhamento dessa base conceitual serviu de suporte para a avaliação de validade conceitual dos instrumentos por identificar sua classificação segundo as subdimensões de violência abarcadas, conforme pormenorizado adiante.

Nesse sentido, optou-se por utilizar a portaria do Ministério da Saúde (MS) do Brasil, de 2001, cujas recomendações são bastante consistentes com as propostas de vários outros órgãos e pesquisadores 11,15. A prerrogativa dessa opção é preservar uma homogeneidade de conceitos no território brasileiro. A portaria estabelece diretrizes para uma política nacional de redução da morbimortalidade por acidentes e violências e descreve várias formas de violência, incluindo as contra o idoso. Nesta seção específica, sete tipos de violências são definidos. O abuso físico ou maus-tratos físicos concerne ao uso de força física, potencialmente produtor de uma injúria, ferida, dor ou incapacidade. Abuso ou maus-tratos psicológicos envolve agressões verbais ou gestuais com o objetivo de aterrorizar, rejeitar, humilhar a vítima, restringindo sua liberdade ou, ainda, isolá-la do convívio social. A negligência é a recusa, omissão ou fracasso por parte do responsável no cuidado com a vítima, porquanto a autonegligência é conceituada como conduta de pessoa idosa que ameaça sua própria saúde ou segurança, com a recusa ou o fracasso de prover a si mesma um cuidado adequado. Abandono é descrito como ausência ou deserção, por parte do responsável, dos cuidados necessários às vítimas, às quais caberia prover custódia física e cuidado. Abuso financeiro aos idosos ocorre quando há exploração imprópria ou ilegal e/ou uso não consentido de seus recursos financeiros. Por fim, o abuso sexual seria o ato ou jogo sexual, em uma relação hétero ou homossexual, visando estimular a vítima ou utilizá-la para obter excitação sexual e práticas eróticas/se- 
xuais impostas por meio de aliciamento, violência física ou ameaças.

A segunda etapa do processo consistiu na identificação dos instrumentos utilizados na detecção de violência doméstica em idosos. Realizou-se uma busca bibliográfica nas bases de dados MEDLINE entre os anos de 1966 e 2004, LILACS entre 1990 e 2004, PsycINFO entre 1984 e 2004, OVIDS citations até 2004 e CANE (Clearinghouse on Abuse and Neglect of The Elderly) também até 2004. Nas três primeiras bases, foram utilizados sete descritores, a saber, elder abuse (domestic violence or family violence); (elder or aged or old or ageing or elderly); (vulnerable or disabled or handicapped); mass screening/or screening; questionnaires/or questionnaire; interview/or interview. Para a busca no LILACS, utilizaram-se os termos equivalentes em português, a saber: violência idosos; (violência doméstica ou violência familiar ou violência íntima); (idoso ou velho ou ancião ou envelhecimento); (frágil ou incapaz ou em desvantagem); rastreamento populacional/ou rastreamento ou screening; questionários/ou questionário ou instrumento; entrevistalou entrevistas. Também foram usados termos equivalentes em espanhol. Na base CANE, que é específica para violência em idosos, utilizaram-se os termos mass screening/or screening; questionnaires/or questionnaire; interview or interview. Além dessas fontes, foram incluídos livros de referência sobre o tema 11,16,17,18,19.

Foram considerados todos os tipos de publicação relativa a estudos realizados em seres humanos e de qualquer faixa etária, pois instrumentos concebidos para uso em situações de violência que não contra os idosos poderiam estar sendo utilizados em faixas etárias mais avançadas. Interessaram os instrumentos produzidos nas línguas inglesa, francesa, espanhola e portuguesa. Foram também identificadas duas revisões recentes sobre o tema, que serviram de norte para a estratégia de busca utilizada e fontes de referências suplementares 20,21.

A terceira etapa processual envolveu uma seleção dos instrumentos de risco em particular, com pesquisa bibliográfica efetuada nas mesmas bases e períodos usados na etapa anterior. Procurou-se escolher instrumentos descritos ou concebidos como instrumentos de avaliação de risco, segundo a classificação proposta por Johnson ${ }^{22}$. O termo risco é utilizado pela autora para designar uma situação de violência potencial ou presente. Dessa forma, entende a autora que a detecção pode ser mais precoce do que quando se avaliam apenas sinais abertos relacionados às conseqüências do abuso ${ }^{23}$. Critérios de seleção adicionais incluí- ram instrumentos a escolher que se aplicassem à prática clínica; que fossem conduzidos em ambientes de atendimento primário de medicina de família, medicina interna geral ou medicina geriátrica; e que incluíssem um médico ou outro agente de saúde no processo de avaliação.

Uma vez escolhidos os instrumentos, a segunda fase da terceira etapa envolveu um escrutínio sistemático dos respectivos históricos psicométricos. Procurou-se, também, apreciar a autoria, o tipo de escala utilizada, o número de itens constituintes, duração de aplicação e o método de administração (se por autopreenchimento, entrevista direta ou proxy). Também se procurou avaliar, em cada instrumento encontrado, as diversas dimensões de violência abarcadas, tomando-se, como base, as definições do MS antes mencionadas 14. Alguns critérios foram estabelecidos para a apreciação dos estudos de validade e confiabilidade, tomando-se, como norma, as diretrizes descritas por Streiner \& Norman 24 e Nunnaly \& Bernstein 25.

No que tange à validade conceitual, procurou-se identificar se havia um conceito ou teoria claramente explicitada pelos proponentes do instrumento. A validade de conteúdo foi também analisada com base nas descrições dos autores de cada instrumento. A validade de face foi aceita caso houvesse menção da utilização de grupos de especialistas na sua avaliação. A adequação da validade de critério baseou-se nos tradicionais estimadores de sensibilidade e especificidade, aceitando-se medidas acima de 0,75 como boas e valores acima de 0,85 como excelentes. A validade de construto foi avaliada pela presença dos diversos procedimentos de análise fatorial 25 , bem como por comparações com dimensões correlatas e/ou fatores de risco conhecidos ou putativos. Procedimentos de análise fatorial exploratória e confirmatória foram identificados. Variáveis salientes em fatores com valores de 0,50 foram consideradas altas 25 . A presença de análises fatoriais por componentes principais e por eixos principais também foi escrutinada.

Nas avaliações formais de concordância em que o estimador kappa foi utilizado, seguiu-se a orientação de Shrout 26 , que propõe a seguinte classificação: ausente se $\kappa<0,10$; fraca se $\kappa=$ 0,10 a 0,40 ; discreta se $\kappa=0,41$ a 0,60 ; moderada se $\kappa=0,61$ a 0,80 ; e substancial se $\kappa>0,80$. Valores de kappa acima de 0,60 foram considerados, portanto, adequados. A qualificação das correlações intraclasse seguiu o mesmo padrão. Para o índice de consistência interna, valores acima de 0,70 foram considerados bons, e valores acima de 0,80 foram considerados muito 
bons 25,27 , embora valores tendendo para a unidade possam significar itens redundantes em uma mesma dimensão avaliada. O coeficiente de Pearson possui alguma limitação no contexto de estudos psicométricos, e estudos que os utilizaram não foram considerados, em princípio, adequados 26 .

Para delinear um quadro de síntese da apreciação dos instrumentos, seguiu-se a orientação de McDowell \& Newell 27 que propõem dois modos de avaliação do histórico de cada instrumento. A completeza concerne uma apreciação sobre o acúmulo e aprofundamento dos estudos de confiabilidade ou validade do instrumento na literatura e pode ser resumida em três níveis. Considera-se basal quando há apenas uma descrição ou conceituação teórica sobre o instrumento, mas inexistem estudos psicométricos ou os artigos correspondentes não descrevem, de forma clara, como o instrumento foi estudado no tocante às suas qualidades psicométricas. O nível é considerado bom quando o histórico psicométrico do instrumento encerra resultados oriundos de vários testes e excelente se todas as formas de avaliação de confiabilidade e validade descritas acima são contempladas simultaneamente.

Como a completeza dos históricos, por definição, independem dos resultados obtidos, o segundo modo de avaliação aprecia a qualidade dos resultados de confiabilidade e validade. Quando não é possível interpretar os resultados a partir dos estudos disponíveis, a situação é indicada por “?” nas respectivas tabelas apresentadas na seção de resultados. A qualificação "0" aponta que, mesmo havendo relato de avaliações psicométricas no histórico de desenvolvimento do instrumento, não se encontrou qualquer estudo fornecendo evidências numéricas nos resultados. Com base nos critérios de adequação relativos à validade de construto, de critério, via análise fatorial ou de confiabilidade descritos anteriormente, avalia-se como insuficiente quando essas propriedades não alcançam resultados satisfatórios em nenhum dos critérios. Por contigüidade, considera-se o histórico adequado quando há evidência de que ao menos uma dessas avaliações psicométricas se encontra dentro dos parâmetros de qualidade ideais estipulados. Por fim, procurou-se identificar se havia, dentre os instrumentos de risco, algum com adaptação transcultural para o meio cultural brasileiro. Também, analisou-se o processo de adaptação, avaliando-se sua adequação segundo critérios sugeridos por alguns autores de referência 28,29,30,31.

\section{Resultados}

As Tabelas 1, 2 e 3 apresentam os 17 instrumentos encontrados ao aplicar-se os filtros de seleção descritos na seção de métodos. A Tabela 1 indica as dimensões que cada instrumento abarca. Observa-se que nenhum avalia autonegligência, e somente dois (Elder Abuse Assessment Tool-EAI-FULMER -, Health Status Risk Assessment-JOHNSON -) exploram itens relacionados a abandono. Três instrumentos Akron General Medical Center Geriatric Abuse Protocol (AKRON), Elder Abuse Assessment Protocol for Nurses (EAAPN) e JOHNSON - possuem itens cobrindo a perpetração de violência sexual. O último é o instrumento com maior validade de conteúdo, ou seja, que avalia mais dimensões. O Risk of Elder Abuse in the Home (REAH), que é dividido em uma seção investigando o idoso e outra o cuidador, propõe-se a inquirir sobre riscos gerais de violência, vulnerabilidade do idoso e sobrecarga do cuidador, não investigando, portanto, dimensões específicas de violência.

A Tabela 2 sintetiza os 81 estudos que foram publicados sobre os 17 instrumentos. Verificase que todos os instrumentos são de língua inglesa, embora alguns sejam de países outros que os Estados Unidos, como, por exemplo, o Brief Abuse for the Elderly (BASE), Caregiver Abuse Screen (CASE), Indicators of Abuse Screen (IOA) do Canadá e o Vulnerability to Abuse Screening Scale (VASS) da Austrália. Excetuando-se a Conflict Tactics Scale (CTS-1) e HwalekSengstock Elder Abuse Screening Test (H-S/ EAST), nenhum outro instrumento foi avaliado fora de sua cultura original. O VASS é resultado da avaliação psicométrica do H-S/EAST, concebido nos Estados Unidos.

Muitos estudos são apenas de apresentação e/ou psicometria, sem ter sido possível identificar qualquer utilização subseqüente. A Tabela 2 também mostra que vários não possuem avaliações psicométricas (AKRON, Carer Abuse Assessment Protocol for Nurses - DAVIES -, EAAPN, High Risk Placement Worksheet - HRPW-KOSBERG -, Screening Protocol for Identification of Abuse and Neglect - SCREENPROT -), embora, eventualmente, possam ser identificadas descrições dos conceitos subjacentes, como mostra a Tabela 3.

Embora a Tabela 2 indique que a CTS- 1 seja o instrumento de utilização mais freqüente, efetivamente, foram encontrados poucos estudos que a tenham usado especificamente em idosos 9,10. De concepção mais antiga, a CTS-1 32 foi, originalmente, criada para avaliar violência íntima em indivíduos mais jovens. Não sur- 
Tipos de violência aferida pelos instrumentos.

\begin{tabular}{|c|c|c|c|c|c|c|c|}
\hline Instrumentos & Física & Psicológica & Negligência & $\begin{array}{l}\text { Violência } \\
\text { Autonegligência }\end{array}$ & Abandono & Financeira & Sexual \\
\hline $\begin{array}{l}\text { Akron General Medical Center Geriatric } \\
\text { Abuse Protocol (AKRON) }\end{array}$ & $\sqrt{ }$ & $\sqrt{ }$ & $\sqrt{ }$ & - & - & $\sqrt{ }$ & $\sqrt{ }$ \\
\hline Brief Abuse Screen for the Elderly (BASE) & $\sqrt{ }$ & $\sqrt{ }$ & $\sqrt{ }$ & - & - & $\sqrt{ }$ & - \\
\hline Caregiver Abuse Screen (CASE) & $\sqrt{ }$ & $\sqrt{ }$ & $\sqrt{ }$ & - & - & $\sqrt{ }$ & - \\
\hline Conflict Tactics Scale (CTS-1) & $\sqrt{ }$ & $\sqrt{ }$ & - & - & - & - & - \\
\hline $\begin{array}{l}\text { Carer Abuse Assessment Protocol } \\
\text { for Nurses (DAVIES) }\end{array}$ & $\sqrt{ }$ & $\sqrt{ }$ & $\sqrt{ }$ & - & - & $\sqrt{ }$ & $\sqrt{ }$ \\
\hline $\begin{array}{l}\text { Elder Abuse Assessment Protocol } \\
\text { for Nurses (EAAPN) }\end{array}$ & $\sqrt{ }$ & $\sqrt{ }$ & $\sqrt{ }$ & - & - & $\sqrt{ }$ & - \\
\hline Elder Abuse Assessment Tool (EAI - FULMER) & $\sqrt{ }$ & - & $\sqrt{ }$ & - & $\sqrt{ }$ & $\sqrt{ }$ & - \\
\hline HALF (HALF) & $\sqrt{ }$ & $\sqrt{ }$ & $\sqrt{ }$ & - & - & $\sqrt{ }$ & - \\
\hline $\begin{array}{l}\text { High Risk Placement Worksheet } \\
\text { (HRPW-KOSBERG) }\end{array}$ & - & - & - & - & - & - & - \\
\hline $\begin{array}{l}\text { Hwalek-Sengstock Elder Abuse Screening } \\
\text { Test (H-S/EAST) }\end{array}$ & $\sqrt{ }$ & $\sqrt{ }$ & $\sqrt{ }$ & - & - & $\sqrt{ }$ & - \\
\hline Fulmer Restriction Scale (FRS) & $\sqrt{ }$ & - & $\sqrt{ }$ & - & - & - & - \\
\hline Indicators of Abuse Screen (IOA) & $\sqrt{ }$ & $\sqrt{ }$ & $\sqrt{ }$ & - & - & $\sqrt{ }$ & - \\
\hline Health Status Risk Assessment (JOHNSON) & $\sqrt{ }$ & $\sqrt{ }$ & $\sqrt{ }$ & - & $\sqrt{ }$ & $\sqrt{ }$ & $\sqrt{ }$ \\
\hline QUALCARE Scale (QUALCARE) & $\sqrt{ }$ & $\sqrt{ }$ & - & - & - & $\sqrt{ }$ & - \\
\hline Risk of Elder Abuse in the Home (REAH) & - & - & - & - & - & - & - \\
\hline $\begin{array}{l}\text { Screening Protocol for Identification } \\
\text { of Abuse and Neglect (SCREENPROT) }\end{array}$ & $\sqrt{ }$ & $\sqrt{ }$ & $\sqrt{ }$ & - & - & $\sqrt{ }$ & - \\
\hline Vulnerability to Abuse Screening Scale (VASS) & $\sqrt{ }$ & $\sqrt{ }$ & $\sqrt{ }$ & - & - & $\sqrt{ }$ & - \\
\hline
\end{tabular}

preende que, mesmo sendo o único instrumento adaptado formalmente para uso no Brasil, sua adaptação tenha se dado em ambiente de violência focalizando faixas etárias aquém dos sessenta anos 33 . Uma outra carência é que a CTS-1 se restringe às dimensões de violência física e psicológica, conforme mostra a Tabela 1.

Como se pode depreender da Tabela 3 (coluna 4), verifica-se que, além da vítima potencial, seis instrumentos também avaliam violência sob a perspectiva do cuidador, que, no caso dos instrumentos encontrados (CTS-1, HALF, IOA, QUALCARE Scale - QUALCARE -, REAH, SCREENPROT), é um agressor em potencial. Dois se restringem ao cuidador como fonte de informações de violência contra o idoso (CASE e DAVIES). A maioria das ferramentas foi concebida para uso em serviços, mas quatro instrumentos (Fulmer Restriction Scale - FRS -, IOA, QUALCARE e REAH) são propostos para utilização em ambiente domiciliar. Vale reparar que, apesar de aparentemente estar-se desrespeitando um dos critérios de inclusão desta revisão, esses foram incluídos porque a avaliação clínica em idosos também pode ser realizada no domicílio ${ }^{34}$. Sete instrumentos utili- zam escalas nominais, porquanto nove encerram escalas ordinais com pontos de corte indicando aumento de risco de violência a partir de certo escore. O QUALCARE e o FRS utilizam escala de Likert. O EAI-FULMER, inicialmente utilizando escala nominal, foi estudado, posteriormente, com escala ordinal 35,36,37.

Ainda na Tabela 3, verifica-se que o IOA é qualificado como bom segundo a completeza. O QUALCARE é o instrumento com melhor perfil de estudos de validade e confiabilidade. A CTS-1, por sua vez, é um instrumento com bom perfil pregresso, embora, deva-se enfatizar novamente, nenhum estudo tenha utilizado população idosa. O CASE, BASE, EAI-FULMER e H-S/EAST possuem poucos estudos com resultados psicométricos satisfatórios. A Tabela 4 oferece uma síntese dos resultados dos sete instrumentos em que houve alguma avaliação mais aprofundada. Em instrumentos como o CASE, EAI-FULMER e IOA, utilizaram-se correlações de Pearson, consideradas inadequadas para avaliar confiabilidade 26 . 
Tabela 2

Instrumentos de violência - características gerais - estudos publicados relacionados.

\begin{tabular}{|c|c|c|c|c|c|c|c|c|c|}
\hline \multirow[t]{2}{*}{ Instrumentos } & \multirow[t]{2}{*}{ Referência } & \multirow[t]{2}{*}{ Ano } & \multirow[t]{2}{*}{$\begin{array}{l}\text { Língua } \\
\text { original }\end{array}$} & \multirow[t]{2}{*}{ Psicometria } & \multirow[t]{2}{*}{$\begin{array}{l}\text { Utilização } \\
\text { internacional }\end{array}$} & \multirow[t]{2}{*}{$\begin{array}{l}\text { Utilização } \\
\text { no Brasil }\end{array}$} & \multirow[t]{2}{*}{$\begin{array}{l}\text { Adaptação } \\
\text { Brasil }\end{array}$} & \multicolumn{2}{|c|}{$\begin{array}{l}\text { Total de } \\
\text { estudos }\end{array}$} \\
\hline & & & & & & & & $\mathrm{n}$ & $\%$ \\
\hline $\begin{array}{l}\text { Akron General Medical Center } \\
\text { Geriatric Abuse Protocol (AKRON) }\end{array}$ & Jones et al. 61 & 1988 & Inglês & 0 & 1 & 0 & 0 & 1 & 1,2 \\
\hline $\begin{array}{l}\text { Brief Abuse Screen for } \\
\text { the Elderly (BASE) }\end{array}$ & $\begin{array}{l}\text { Reis \& } \\
\text { Nahmiash } 62\end{array}$ & 1998 & Inglês & 1 & 2 & 0 & 0 & 3 & 3,7 \\
\hline Caregiver Abuse Screen (CASE) & $\begin{array}{l}\text { Reis \& } \\
\text { Nahmiash } 53\end{array}$ & 1995 & Inglês & 1 & 1 & 0 & 0 & 2 & 2,5 \\
\hline Conflict Tactics Scale (CTS-1) & Straus 32 & 1979 & Inglês & 23 & 18 & 3 & 1 & 45 & 55,5 \\
\hline $\begin{array}{l}\text { Carer Abuse Assessment } \\
\text { Protocol for Nurses (DAVIES) }\end{array}$ & Davies 63 & 1997 & Inglês & 0 & 1 & 0 & 0 & 1 & 1,2 \\
\hline $\begin{array}{l}\text { Elder Abuse Assessment } \\
\text { Protocol for Nurses (EAAPN) }\end{array}$ & Davies 64 & 1993 & Inglês & 0 & 1 & 0 & 0 & 1 & 1,2 \\
\hline $\begin{array}{l}\text { Elder Abuse Assessment } \\
\text { Tool (EAl - FULMER) }\end{array}$ & Fulmer 35 & 1984 & Inglês & 2 & 1 & 0 & 0 & 3 & 3,7 \\
\hline Fulmer Restriction Scale (FRS) & Fulmer 65 & 1996 & Inglês & 1 & 1 & 0 & 0 & 2 & 2,5 \\
\hline HALF (HALF) & $\begin{array}{l}\text { Ferguson \& } \\
\text { Beck } 66\end{array}$ & 1983 & Inglês & 1 & 0 & 0 & 0 & 1 & 1,2 \\
\hline $\begin{array}{l}\text { High Risk Placement Worksheet } \\
\text { (HRPW-KOSBERG) }\end{array}$ & Kosberg 8 & 1988 & Inglês & 0 & 1 & 0 & 0 & 1 & 1,2 \\
\hline $\begin{array}{l}\text { Hwalek-Sengstock Elder Abuse } \\
\text { Screening Test (H-S/EAST) }\end{array}$ & $\begin{array}{l}\text { Hwalek \& } \\
\text { Sengstock } 55\end{array}$ & 1986 & Inglês & 5 & 2 & 0 & 0 & 7 & 8,6 \\
\hline Indicators of Abuse Screen (IOA) & $\begin{array}{l}\text { Reis \& } \\
\text { Nahmiash } 56\end{array}$ & 1998 & Inglês & 2 & 4 & 0 & 0 & 6 & 7,4 \\
\hline $\begin{array}{l}\text { Health Status Risk Assessment } \\
\text { (JOHNSON) }\end{array}$ & Johnson 22 & 1991 & Inglês & 1 & 1 & 0 & 0 & 2 & 2,5 \\
\hline QUALCARE Scale (QUALCARE) & $\begin{array}{l}\text { Phillips } \\
\text { et al. } 57,58\end{array}$ & 1996 & Inglês & 2 & 0 & 0 & 0 & 2 & 2,5 \\
\hline $\begin{array}{l}\text { Risk of Elder Abuse } \\
\text { in the Home (REAH) }\end{array}$ & Hamilton 67 & 1989 & Inglês & 1 & 0 & 0 & 0 & 1 & 1,2 \\
\hline $\begin{array}{l}\text { Screening Protocol for Identification } \\
\text { of Abuse and Neglect (SCREENPROT) }\end{array}$ & Johnson 16 & 1981 & Inglês & 0 & 1 & 0 & 0 & 1 & 1,2 \\
\hline $\begin{array}{l}\text { Vulnerability to Abuse } \\
\text { Screening Scale (VASS) }\end{array}$ & $\begin{array}{l}\text { Schofield \& } \\
\text { Mishra } 50\end{array}$ & 2002 & Inglês & 2 & 0 & 0 & 0 & 2 & 2,5 \\
\hline Total & & & & 42 & 35 & 3 & 1 & 81 & 100,0 \\
\hline
\end{tabular}

\section{Discussão}

A recuperação e/ou manutenção da qualidade de vida vem sendo apontada como um dos objetivos centrais na abordagem clínica de pacientes idosos fragilizados. A história, o exame físico e o diagnóstico diferencial tradicionais não são suficientes para um levantamento amplo das diversas funções físicas, psicológicas e sociais necessárias à vida diária. Uma vez preocupada com a qualidade de vida, a prática clínica geriátrica deve contemplar uma ampla avaliação funcional em busca de perdas possíveis nessas áreas. Os princípios básicos da gerontologia buscam a promoção da saúde das pessoas idosas. Visam à diminuição da incidência de declínio funcional através da pre- venção de doenças, de suas seqüelas e complicações, assim como a manutenção da capacidade funcional com o intuito de preservar independência e autonomia. Essa perspectiva também oferece, aos profissionais da área, a possibilidade de identificação da ocorrência de atos violentos e a elaboração de estratégias de intervenção adequadas para manter a pessoa idosa protegida. Ademais, isso pode ser realizado em um ambiente de cuidados de saúde, dado que os indivíduos idosos tendem a procurar serviços mais amiúde do que pessoas jovens 20.

Há algum tempo, o atendimento do paciente geriátrico já é orientado para a avaliação interdisciplinar dos distúrbios funcionais. Tornase, portanto, natural incorporar a avaliação e 
Psicometria de instrumentos de violência.

\begin{tabular}{|c|c|c|c|c|c|c|c|c|c|c|c|}
\hline \multirow[t]{2}{*}{ Instrumentos } & \multirow[t]{2}{*}{ Escala } & \multirow{2}{*}{$\begin{array}{l}\text { Número } \\
\text { de itens }\end{array}$} & \multirow{2}{*}{$\begin{array}{l}\text { Grupo } \\
\text { avaliado }\end{array}$} & \multirow{2}{*}{$\begin{array}{l}\text { Local } \\
\text { de apli- } \\
\text { cação }\end{array}$} & \multirow{2}{*}{$\begin{array}{l}\text { Adminis- } \\
\text { trado por } \\
\text { (tempo) }\end{array}$} & \multicolumn{3}{|c|}{ Confiabilidade } & \multicolumn{3}{|c|}{ Validade } \\
\hline & & & & & & $\begin{array}{l}\text { Tipo de } \\
\text { análise }\end{array}$ & $\begin{array}{l}\text { Comple- } \\
\text { teza }\end{array}$ & $\begin{array}{l}\text { Qualifi- } \\
\text { cação dos } \\
\text { resultados }\end{array}$ & $\begin{array}{l}\text { Tipo de } \\
\text { s análise } \\
\text { s }\end{array}$ & $\begin{array}{l}\text { Comple- } \\
\text { teza }\end{array}$ & $\begin{array}{l}\text { Qualifi- } \\
\text { cação dos } \\
\text { resultados }\end{array}$ \\
\hline $\begin{array}{l}\text { Akron General Medical Center } \\
\text { Geriatric Abuse Protocol (AKRON) }\end{array}$ & Nom & 46 & Vit & Cli & $\mathrm{S}$ & - & 0 & $\mathrm{Sn}$ & - & 0 & $\mathrm{Sn}$ \\
\hline $\begin{array}{l}\text { Brief Abuse Screen } \\
\text { for the Elderly (BASE) }\end{array}$ & Ord & 5 & Vit & Tri & $\mathrm{S}$ & Inter, intra & Bas & Adeq & VC, Cns & Bas & Adeq \\
\hline Caregiver Abuse Screen (CASE) & Ord & 8 & Cuid & Tri & $\begin{array}{c}\mathrm{SS} \\
(1-2 \mathrm{~min})\end{array}$ & $\mathrm{Ci}$ & Bas & Adeq & VC, $\mathrm{F}, \mathrm{Cc}$ & Bas & Adeq \\
\hline Conflict Tactics Scale (CTS-1) & Ord/Lik & 19 & Vit/Cuid & Cli & $\begin{array}{c}\mathrm{S} \\
(7-10 \mathrm{~min})\end{array}$ & $\begin{array}{l}\mathrm{Ci} \text {, intra, } \\
\text { inter }\end{array}$ & Boa & Adeq & $\begin{array}{l}\text { VC, Cnt, } \\
\text { D, Cns }\end{array}$ & Boa & Adeq \\
\hline $\begin{array}{l}\text { Carer Abuse Assessment } \\
\text { Protocol for Nurses (DAVIES) }\end{array}$ & Nom & 41 & Cuid & Cli & S & - & 0 & Sn & - & 0 & Sn \\
\hline $\begin{array}{l}\text { Elder Abuse Assessment } \\
\text { Protocol for Nurses (EAAPN) }\end{array}$ & Nom & 80 & Vit & $\mathrm{Cli} / T r i$ & $\mathrm{~S}$ & - & 0 & $\mathrm{Sn}$ & - & 0 & $\mathrm{Sn}$ \\
\hline $\begin{array}{l}\text { Elder Abuse Assessment } \\
\text { Tool (EAI - FULMER) }\end{array}$ & Nom/Ord & 44 & Vit & Tri & $\begin{array}{c}\mathrm{S} \\
(12-15 \mathrm{~min})\end{array}$ & Inter, $\mathrm{Ci}$ & Bas & Adeq & $\mathrm{Cc}, \mathrm{Cnt}$ & Bas & $\mathrm{Sn}$ \\
\hline Fulmer Restriction Scale (FRS) & Ord/Lik & 34 & Vit & Clidom & S & - & 0 & $\mathrm{Sn}$ & VC, Cns & Bas & Insuf \\
\hline HALF (HALF) & Nom & 37 & Vit/Cuid & Tri & $\mathrm{S}$ & - & 0 & $\mathrm{Sn}$ & VC & 0 & $\mathrm{Sn}$ \\
\hline $\begin{array}{l}\text { High Risk Placement Worksheet } \\
\text { (HRPW-KOSBERG) }\end{array}$ & Nom & 40 & Vit/Cuid & Cli & - & - & 0 & Sn & VC & 0 & Sn \\
\hline $\begin{array}{l}\text { Hwalek-Sengstock Elder Abuse } \\
\text { Screening Test (H-S/EAST) }\end{array}$ & Ord & 15 & Vit & Tri & $\begin{array}{c}\text { S-A } \\
(5-10 \mathrm{~min})\end{array}$ & $\mathrm{Ci}$ & Bas & Insuf & $\begin{array}{l}\text { VC, F, Cnt, } \\
\text { D, Cns }\end{array}$ & Boa & Adeq \\
\hline Indicators of Abuse Screen (IOA) & Ord & 22 & Vit & Clidom & $\begin{array}{c}\text { SS } \\
(20 \mathrm{~min})\end{array}$ & $\mathrm{Ci}$ & Bas & Adeq & $\begin{array}{l}\text { VC, F, } \\
D, C n s\end{array}$ & Boa & Adeq \\
\hline $\begin{array}{l}\text { Health Status Risk Assessment } \\
\text { (JOHNSON) }\end{array}$ & Nom & 60 & Vit & Tri & S & - & 0 & $\mathrm{Sn}$ & VC & 0 & Sn \\
\hline QUALCARE Scale (QUALCARE) & Ord/Lik & 40 & Vit/Cuid & Clidom & $\mathrm{S}$ & Inter, $\mathrm{Ci}$ & Boa & Adeq & $\begin{array}{l}\text { VC, F, Cnt, } \\
\text { D, Cc, } \\
\text { Cns, FACE }\end{array}$ & Exc & Adeq \\
\hline $\begin{array}{l}\text { Risk of Elder Abuse } \\
\text { in the Home (REAH) }\end{array}$ & Ord & 34 & Vit/Cuid & Clidom & SS & - & 0 & $\mathrm{Sn}$ & VC, Cnt, D & Bas & Insuf \\
\hline $\begin{array}{l}\text { Screening Protocol for } \\
\text { Identification of Abuse } \\
\text { and Neglect (SCREENPROT) }\end{array}$ & Nom & 38 & Vit/Cuid & $\mathrm{Cli}$ & $\mathrm{S}$ & - & 0 & $\mathrm{Sn}$ & - & 0 & $\mathrm{Sn}$ \\
\hline $\begin{array}{l}\text { Vulnerability to Abuse Screening } \\
\text { Scale (VASS) }\end{array}$ & Ord & 12 & Vit & Tri & A & $\mathrm{Ci}$ & Bas & Insuf & $\begin{array}{l}\text { VC, F, } \\
\text { Cnt, D }\end{array}$ & Bas & Adeq \\
\hline
\end{tabular}

Ord = Ordinal (Lik = Likert); Nom = Nominal; Vit = vítima idosa ou em potencial; Cuid = cuidador do idoso ou potencial agressor; Vit/Cuid = seções de avaliação da vítima e cuidador no mesmo instrumento; $\mathrm{Cli}=$ avaliação clínica de violência; Tri = Triagem clínica; Clidom = uso em avaliação domiciliar de triagem ou avaliação clínica; $\mathrm{S}=$ Staff; $\mathrm{A}=$ autopreenchimento; SS = serviço social; intra = confiabilidade intra-observador; inter = confiabilidade interobservador; $\mathrm{Ci}=$ consistência interna; $\mathrm{F}=$ análise fatorial; $\mathrm{D}=$ validade de critério, tipo discriminante; $\mathrm{Pr}=$ validade de critério, tipo preditiva; $\mathrm{Cc}=$ validade de critério, tipo concorrente; $\mathrm{VC}=$ validade conceitual; $\mathrm{Cnt}=$ validade de conteúdo; $\mathrm{FACE}=$ validade de face; $\mathrm{Cns}=$ validade de construto, sem especificação; $0=$ sem evidência de confiabilidade ou validade; Bas = informação apenas basal; Boa = vários tipos de teste ou vários estudos: Exc = todas as principais formas de teste para confiabilidade/validade descritas; $\mathrm{S} n=$ sem resultados numéricos; Insuf = confiabilidade/ validade construto, critério ou análise fatorial insuficientes; Adeq = confiabilidade/validade construto, critério ou análise fatorial adequadas (definição, vide texto).

atendimento do idoso vítima ou envolvido em atos violentos nessa abordagem geriátrica geral. A importância dos distúrbios funcionais associados à violência pode ser explorada em um ambiente que já avalia, sistematicamente, o status funcional 38. A inserção de avaliação de suspeitas de violência contra o idoso em um ambiente geriátrico de avaliação interdisciplinar, como o é na Avaliação Geriátrica Ampla
$20,34,39$, traria suporte maior para que se cumprisse o objetivo primordial do profissional interessado pelos problemas do envelhecimento. Manter a independência do indivíduo idoso e sua qualidade de vida acarretaria uma postergação máxima de sua incapacidade, se possível, até um período próximo à sua morte, no que se denomina compressão de morbidade. Percebe-se, portanto, que a avaliação da vio- 
Instrumentos de violência. Síntese dos resultados numéricos.

\begin{tabular}{|c|c|}
\hline Instrumentos & Achados \\
\hline Brief Abuse Screen for the Elderly (BASE) & $r=0,86-0,9$ \\
\hline Caregiver Abuse Screen (CASE) & $\begin{array}{l}\text { Escores distinguiram agressores de não agressores }(\mathbf{C i} \alpha=0,71) \text {. Outras características } \\
\text { semelhantes. } \\
\text { Correlações com outros instrumentos: Ryden } r=0,4 \text { e } 0,25 ; \text { IOA } r=0,41 \text {; } \\
\text { Indicadores de cuidadores } r=0,40 ; \text { Indicadores dos idosos } r=0,37 ; \mathrm{H}-\mathrm{S} / \text { EAST } r=0,26 \text {. }\end{array}$ \\
\hline Conflict Tactis Scale (CTS-1) & $\mathrm{Ci} \alpha=0,88 ; \mathrm{Cnt}=0,80 ; \kappa>0,70 ; \mathbf{D} 4$ fatores; Cns com vários índices correlatos e diferentes. \\
\hline Elder Abuse Assessment Tool (EAI-FULMER) & Ci $\alpha=0,84 ; r=0,83-0,889 ; \kappa=0,65 ;$ sens $=0,71 ;$ esp $=0,93$ \\
\hline $\begin{array}{l}\text { Hwalek-Sengstock Elder Abuse Screening } \\
\text { Test (H-S/EAST) }\end{array}$ & $\begin{array}{l}\text { Escores distinguiram vítimas de não vítimas }(\alpha=0,29) \text {. Seis itens relacionados fortemente } \\
\text { com abuso } \\
\text { F: } 3 \text { itens. }\end{array}$ \\
\hline Indicators of Abuse Screen (IOA) & $\begin{array}{l}\text { Ci } \alpha=0,92 \text {; Sens }=0,844 \text {; esp }=0,992 ; r=0,37-0,41, \mathbf{D}=0,80 \text {. Escores para vítimas e não } \\
\text { vítimas significativamente diferentes }(p=0,049) \text {; classificação correta de } 71 \% \text { dos casos; } \\
\text { discriminou casos de abuso } 84,4 \% \text { das vezes e casos de não abuso } 99,2 \% \text { das vezes. }\end{array}$ \\
\hline QUALCARE Scale (QUALCARE) & $\begin{array}{l}\text { Cnt: técnica Q-sort; inter: } 54 \% \text { subescala psicológica, } 66 \% \text { subescala direitos humanos; } \\
\text { técnica Delphi para FACE; } \kappa / \kappa_{\max } \text { para inter entre } 79 \% \text { e } 88 \% ; 0,65 \text { para a escala total; } \\
\alpha=0,97 ; r \text { inter - subescalas entre } 0,50 \text { e } 0,70 ; F=\text { seis fatores como predito; } 13 \text { itens } \\
\text { excluídos; relação bivariada de } 8 \text { variáveis de critério na direção correta; } C \text { ns = comparação } \\
\text { com nove variáveis com } 8 \text { variáveis na direção predita. }\end{array}$ \\
\hline Vulnerability to Abuse Screening Scale (VASS) & $\begin{array}{l}\alpha=0.55,0.52,0.43,0.39 ; \mathrm{F}: 4 \text { itens respondendo por } 50 \% \text { da variância total. Os } 4 \text { fatores } \\
\text { correlacionados com várias medidas correlatas para } 12.340 \text { mulheres entre } 70 \text { e } 75 \text { anos. }\end{array}$ \\
\hline
\end{tabular}

Tipo de análise: inter = confiabilidade interobservador $\mathbf{C i}=$ consistência interna; $\mathbf{F}=$ análise fatorial; $\mathbf{D}=$ validade de critério,

tipo discriminante; $\mathbf{P r}=$ validade de critério, tipo preditiva; $\mathbf{C c}=$ validade de critério, tipo concorrente; $\mathbf{V C}=$ validade conceitual;

$\mathbf{C n t}=$ validade de conteúdo; FACE = validade de face; $\mathbf{C n s}=$ validade de construto, sem especificação; sens = sensibilidade;

esp = especificidade; $\alpha=$ índice de consistência interna de Cronbach (alfa); $\kappa=$ índice kappa de reprodutibilidade.

lência, de cunho interdisciplinar, possui pontos comuns com a Avaliação Geriátrica Ampla. Valeria utilizar sua estrutura ao inserir-se essa emergente questão na avaliação geral do indivíduo idoso com risco de distúrbios funcionais que, a rigor, também incorre em elevado risco de abusos e negligência 40,41,42,43.

Em geral, fora do ambiente de emergência, o profissional de saúde se depara mais freqüentemente com formas sutis de violência nas quais a negligência e/ou o abuso psicológico predominam 38. A identificação dessas apresentações mais insidiosas de abuso é dificultada pela presença concomitante de doenças crônicas em idosos, cujas manifestações podem mimetizar atos violentos ou diminuir a suspeição clínica dos mesmos. A avaliação estruturada de risco ou suspeita de violência através da utilização de instrumentos de rastreamento, certamente, pode facilitar a tarefa.

Apesar da ênfase dada à importância da utilização de instrumentos para a detecção de abuso em idosos 9,10,44,45,46, não há protocolos ou instrumentos universalmente aceitos para a triagem ou avaliação da violência doméstica nessa população 47 . Segundo Rosenblatt 48 , isso se explica pela complexidade do problema. As definições sobre abuso diferem, por vezes, em um mesmo país. A raridade de pesquisas na área de violência contra idosos tem como conseqüência o parco conhecimento sobre os fatores de risco correlatos e a pouca clareza sobre os marcadores de violência que devem ser identificados através dos instrumentos de rastreamento e de avaliação.

O abuso em idosos vem em múltiplas formas, cujas conseqüências freqüentemente se sobrepõem e se confundem com sinais e sintomas relacionados a diversas patologias prevalentes nessa faixa etária 48 . Alguns autores ponderam que a utilização de instrumentos de maneira isolada não proporciona uma avaliação adequada em idosos 49. Porém, os instrumentos se constituem em avaliações estruturadas que servem de base para a avaliação sistemática de riscos de violência. 
A identificação da violência em idosos é um processo relativamente novo. Por esse motivo, as medidas de identificação mencionadas na literatura variam desde listas não sistematizadas de indícios de atos violentos até métodos uniformizados. A vantagem das últimas é a possibilidade de replicação dos dados. Vários autores sugerem que a avaliação de violência no sujeito idoso deva armar-se de documentação estruturada visando, além de uma análise mais organizada do nível de saúde, a um melhor preparo para encaminhamentos processuais e legais 5,45,50. Ademais, a entrevista de acompanhantes ou cuidadores, presentes na maior parte das vezes no convívio com o idoso, complementa a informação conseguida através da avaliação das próprias vítimas em potencial.

Este estudo oferece um levantamento dos instrumentos de avaliação e suspeita de violência, com ênfase nessa última, por conta do ambiente clínico ambulatorial não especializado em que se pretende que os instrumentos sejam inseridos. Na atual revisão, procurou-se excluir protocolos muito genéricos ou de avaliação eminentemente qualitativa. Revisões recentes identificaram uma quantidade menor de instrumentos e correlatos estudos publicados, possivelmente por usarem critérios de inclusão mais rígidos ou mecanismos de busca menos exaustivos 20,21,51. Uma indicação disso é que, nessa literatura, não há referência à busca em bases de dados específica para violência em idosos como a CANE.

Os instrumentos encontrados na presente revisão não possuem, individualmente, um grande número de publicações sobre os respectivos estudos de desenvolvimento e utilização. Também, não foi possível identificar qualquer estudo no âmbito da comunidade científica brasileira que se debruçasse sobre o aprimoramento de instrumentos para uso no país, quer mediante adaptações transculturais formais de ferramentas já empregadas alhures ou mesmo através do desenvolvimento de novas. Vale insistir que a adaptação da CTS-1 para o contexto brasileiro não focalizou a terceira idade 33 .

A atual revisão não encontrou, nas bases de dados mencionadas, qualquer publicação de concepção ou utilização de instrumentos de violência em idosos em português, espanhol ou francês. Não chega a surpreender que a totalidade tenha sido concebida em língua inglesa, uma vez que o interesse e a pesquisa na área de violência doméstica estejam presentes há mais tempo nos Estados Unidos e Reino Unido 17. Há, entretanto, muitos hiatos nas evidências concernentes às qualidades psicomé- tricas desses instrumentos. A Tabela 1 indica, por exemplo, a pouca especificidade de vários instrumentos no que concerne à avaliação das diversas subdimensões da violência. Esse é o caso do HRPW-KOSBERG e REAH. Adicionalmente, depreende-se das Tabelas 3 e 4 que os processos de investigação dos instrumentos encontram-se, ainda, em estágios iniciais. Há poucos estudos psicométricos nas culturas originais, raros estudos são aqueles enfocando adaptações transculturais e ainda falta uma continuidade dos processos de investigação a muitos instrumentos escrutinados. Excetuando-se a CTS-1 e H-S/EAST, nenhum outro foi avaliado fora de sua cultura original. Desenvolvido na Austrália, o VASS é resultado da avaliação psicométrica do H-S/EAST, concebido nos Estados Unidos.

A despeito dessa aparente insuficiência, instrumentos como o BASE, CASE, EAI-FULMER, H-S/EAST, IOA, QUALCARE e VASS possuem algumas boas propriedades psicométricas e poderiam ser mais bem explorados no contexto da avaliação clínica de violência em idosos no Brasil. O BASE é um instrumento rápido, com cinco perguntas breves e duração de um minuto apenas. Sua vantagem é poder ser utilizado em ambientes clínicos com grande movimento, como, por exemplo, serviços de emergência 52. Seu histórico de investigação, porém, resume-se a um único artigo psicométrico publicado que indica boa validade conceitual e uma satisfatória confiabilidade com concordâncias interobservadores variando de $86 \%$ a $90 \%$. Seriam necessários outros estudos avaliando validade de construto, critério, consistência interna e uma maior completeza dos estudos psicométricos.

O CASE é o único instrumento de coleta de informações específicas do cuidador, avaliando comportamentos relacionados a atos violentos por parte deste em relação ao idoso. A saúde e circunstâncias de vida dos cuidadores estão, com freqüência, intimamente ligadas àquelas das vítimas, e a experiência clínica sugere que o reconhecimento da díade cuidadoridoso e a intervenção direta nesse relacionamento oferecem mais possibilidades de quebrar-se um eventual ciclo de violência em curso 20. O CASE não procura abarcar possíveis justificativas ou racionalizações para o ato violento 53. Contém itens que indagam ao cuidador sobre situações que poderiam indicar violência física, psicossocial, material e negligência, sem argüir, explicitamente, sobre comportamentos diretamente relacionados à violência. Pela sua brevidade e simplicidade, o instrumento pode ser utilizado, segundo Reis \& 
Nahmiash 53, em pesquisas como uma ferramenta de rastreamento inicial de violência, valendo-se, inclusive, de entrevistadores sem muita experiência no tema. Na prática clínica, pode servir como um primeiro alerta, talvez em combinação com outros instrumentos de captação. De uma perspectiva operacional, as autoras identificaram escores de quatro ou mais como indicativos ou sugestivos de maior risco.

Junto como o QUALCARE, a CTS-1 e o EAIFULMER parecem ser os mais robustos sob o aspecto psicométrico, como sintetizam as Tabelas 3 e 4 . Entretanto, a CTS-1 possui limitações importantes no que concerne a seu escopo, pois explora apenas violência física e psicológica. Ademais, não existem, ainda, estudos de validade e confiabilidade na população idosa, conforme apontado anteriormente. Por sua vez, a despeito de ter sido concebido para utilização em idosos em ambiente clínico e possuir boas qualidades psicométricas, o EAI-FULMER requer treinamento especial na área de violência para que possa ser adequadamente utilizado. Seus itens são dependentes de avaliação e interpretação por parte do entrevistador, além de conhecimento da história pregressa familiar e social do indivíduo entrevistado. Isso o torna um instrumento dependente de informações que nem sempre estão facilmente disponíveis em ambientes ambulatoriais e hospitalares.

O H-S/EAST é um instrumento breve desenvolvido nos Estados Unidos, resultado de um esforço na identificação de indicadores e sintomas de abuso em idosos. A identificação dos correlatos de abuso teria grande interesse na concepção de um instrumento de rastreamento, pois poderiam estar presentes anteriormente ao evento violento. No seu desenvolvimento, foram testados mais de mil itens selecionados a partir de vários protocolos em uso nos Estados Unidos. Muitos desses itens não focalizavam sintomas específicos de abuso ou negligência, mas tinham a intenção de detectar circunstâncias correlatas à presença do problema como, por exemplo, dependência física ou financeira e isolamento 54 . Uma análise fatorial dos 15 itens do H-S/EAST identificou três categorias conceituais, a saber, violação aberta dos direitos pessoais ou abuso direto; características do idoso que o tornam mais vulnerável ao abuso; e características de uma situação de abuso potencial 55. Estudos de validação também corroboraram sua capacidade para captar possibilidade de abuso psicológico, físico, violação de direitos pessoais e abuso financeiro 54 . Neale et al. 54 advertem que o instrumento deve ser utilizado para identificar suspeita de abuso, particularmente quando o investigador for relativamente pouco experiente no tema. Além das questões concernentes à violência, o H-S/EAST pode ser utilizado para a identificação de serviços que os respondentes possam necessitar, tais como transporte, cuidados pessoais, ou aconselhamento referente ao mau uso de substâncias 54 . O instrumento também serve para decidir-se sobre um aprofundamento na investigação ou encaminhamento do relato de abuso a uma agência especializada 54 .

O IOA é um instrumento relativamente simples, concebido no Canadá, que avalia tanto o cuidador quanto o idoso 56. Deve ser completado por profissionais após uma avaliação domiciliar cuja duração chega a 2 ou 3 horas. Composto de 27 itens referentes a problemas relativos ao idoso ou seu cuidador, indaga sobre a dependência econômica deste em relação ao idoso, problemas passados que tenham relação com alguma forma de violência e dificuldades de relacionamento interpessoal. O instrumento possui boa validade de face e de conteúdo, sendo, entretanto, algo longo para utilização em ambientes clínicos mesmo que domiciliares. Além disso, é necessário treinamento especial dos entrevistadores na área de violência.

O QUALCARE é o mais robusto entre os instrumentos identificados nesta revisão. Concebido nos Estados Unidos para uso de profissionais de enfermagem na avaliação de idosos e seus cuidadores em seus próprios domicílios, avalia seis dimensões, quais sejam, violência física, cuidados médicos, psicossocial, ambiental, direitos humanos e financeira. Segundo Phillips et al. 57,58, são necessárias duas visitas domiciliares com duração total de até duas horas e meia para coletar todas as informações e preencher os quarenta itens do instrumento. A concepção, organização e avaliação psicométrica do QUALCARE são detalhadas e completas, e os resultados bastante promissores. Ainda assim, a duração e a necessidade de treinamento intensivo dos profissionais tornam o instrumento complexo e relativamente custoso.

Concebido na Austrália, o VASS foi avaliado em uma amostra de 12 mil sujeitos, representativa da população de mulheres idosas. A validade de construto foi examinada através de correlações com variáveis sócio-demográficas, psicológicas e relacionadas à saúde, bem como via análise fatorial. Do H-S/EAST original, quatro fatores foram identificados representando as dimensões de abatimento, coerção, dependência e vulnerabilidade, mas 5 dos 17 itens acabaram sendo excluídos. Um item de agressão psicológica, oriundo da CTS-1, foi adicio- 
nado pelas autoras do VASS por possuir alta validade de face. Um segundo item indagando sobre a sensação de medo de alguém na família foi adaptado a partir de uma revisão de vários estudos de fatores correlatos de violência 59.

Em síntese, os instrumentos mais promissores e que merecem uma adaptação para uso no Brasil parecem ser o CASE, EAI-FULMER, H-S/EAST, IOA, QUALCARE e VASS. A escolha dependerá, certamente, da situação na qual se pretende utilizar o ferramental de suspeita. No caso de ambientes clínicos contendo profissionais com treinamento apenas elementar, prioridade poderia ser dada ao CASE, H-S/EAST e VASS. Estratégias de adaptação cultural como, por exemplo, de Herdman et al. 29,60 ou Guillemin et al. 28 devem ser observadas para que se garanta, no Brasil, a disponibilidade de instrumentos eficazes e eficientes de avaliação de violência contra o idoso.

\section{Resumo}

Os dados sobre violência contra o idoso são escassos no Brasil. Para estudos adequados sobre a magnitude, fatores de risco e cuidados às vítimas, é necessária documentação sistemática e acurada. Este artigo revisou os instrumentos de rastreamento e avaliação de violência no idoso visando examinar o progresso no contexto internacional e brasileiro. Utilizando as bases de dados MEDLINE, PsycINFO, LILACS, CANE, OVIDs citations e livros de referência, realizou-se uma revisão sistemática da literatura para a seleção dos instrumentos. Fez-se, também, um escrutínio dos estudos de psicometria e utilização dos instrumentos, aplicando-se critérios para avaliar a qualidade dos estudos. Dos dezessete instrumentos encontrados, somente a Conflicts Tactics Scale foi adaptada para o Brasil, ainda que para uso em um grupo etário mais jovem. Alguns outros com boas características psicométricas também foram encontrados, como o Caregiver Abuse Screen, Elder Abuse Assessment Tool, Hwalek Sengstok Abuse Screening Test, Indicators of Abuse Screen, Qualcare scale e Vulnerability to Abuse Screening Scale. Ainda assim, de forma geral, há lacunas importantes nos processos de concepção, validação e adaptação desses instrumentos no Brasil e no exterior.

\section{Colaboradores}

C. M. Paixão Jr. participou das seguintes etapas: escolha do tema, organização do trabalho, montagem das tabelas de revisão, procura e revisão das publicações, escolha dos instrumentos para revisão, discussão, organização do artigo, revisão e edição final do artigo. M. E. Reichenheim participou das etapas: escolha do tema, organização do trabalho, discussão, organização e correção do artigo, revisão e edição final do artigo.

\section{Agradecimentos}

C. M. Paixão Jr. foi apoiado pela Fundação Carlos Chagas Filho de Amparo à Pesquisa do Estado do Rio de Janeiro, processo n. E-26/150.727/2002. M. E. Reichenheim foi parcialmente apoiado pelo Conselho Nacional de Desenvolvimento Científico e Tecnológico, processo n. 300234/94-5. 


\section{Referências}

1. Barnett O, Miller-Perrin C, Perrin RD. History and definitions. In: Barnett O, Miller-Perrin C, Perrin RD, editors. Family violence across the lifespan: an introduction. London: Sage Publications; 1997. p. 3-17.

2. Gelles RJ. Introduction. In: Gelles RJ, editor. Intimate violence in families. New York: Sage Publications; 1997. p. 1-17.

3. Barnett OW, Miller-Perrin CL, Perrin RD. Introduction. In: Barnett OW, Miller-Perrin CL, Perrin RD, editors. Family violence across the lifespan: an introduction. Thousand Oaks: Sage Publications; 1997. p. XIX-XX.

4. Glendenning F. Attitudes to older people. In: Decalmer P, Glendenning F, editors. The mistreatment of elderly people. London: Sage Publications; 1997. p. 13-41.

5. Machado L, Queiroz ZV. Negligência e maus-tratos. In: Freitas EV, Py L, Neri AL, Cançado FZX, Gorzoni ML, Rocha SM, organizadores. Tratado de geriatria e gerontologia. Rio de Janeiro: Guanabara Koogan; 2002. p. 791-7.

6. Drumond Jr. M, Lira MMTA, Freitas M, Nitrini TMV, Shibao K. Avaliação da qualidade das informações de mortalidade por acidentes não especificados e eventos com intenção indeterminada. Rev Saúde Pública 1999; 33:273-80.

7. Queiroz ZPV. Violência contra a velhice: considerações preliminares sobre uma nova questão social. Mundo Saúde 1997; 21:204-7.

8. Kosberg JI. Preventing elder abuse: identification of high risk factors prior to placement decisions. Gerontologist 1988; 28:43-50.

9. Pillemer KA, Finkelhor D. The prevalence of elder abuse: a random sample survey. Gerontologist 1988; 28:51-7.

10. Podineks E. National Survey on Abuse of the elderly in Canada. Journal of Elder Abuse \& Neglect 1992; 4:5-58.

11. Krug EG, Dahlberg LL, Mercy JA, Zwi AB, Lozano R. World report on violence and health. Geneva: World Health Organization; 2002.

12. American Medical Association Council on Scientific Affairs. Diagnostic and Treatment guidelines concerning child abuse and neglect. Chicago: American Medical Association; 1984.

13. Rathbone-McCuan E, Voyles B. Case detection of abused elderly parents. Am J Psychiatry 1982; 139:189-92.

14. Brasil. Portaria GM/MS n. 737, Política Nacional de Redução da Morbimortalidade por Acidentes e Violências, de 16 de maio de 2001. Diário Oficial da União 2001; 18 mai.

15. Glendenning F. What is elder abuse and neglect? In: Decalmer P, Glendenning F, editors. The mistreatment of elderly people. London: Sage Publications; 1997. p. 13-41.

16. Johnson D. Abuse of the elderly. Nurse Pract 1981; 6:29-34.

17. Barnett OW, Miller-Perrin CL, Perrin RD, editors. Family violence across the lifespan: an introduction. London: Sage Publications; 1997.

18. Decalmer P, Glendenning F. The mistreatment of elderly people. London: Sage Publications; 1997.
19. Tinker A, McCreadie C. Elder abuse. In: Tallis R, Fillit H, Brocklehurst JC, editors. Textbook of geriatric medicine and gerontology. London: Churchill Livingstone; 1998. p. 1431-7.

20. Fulmer T, Guadagno L, Dyer CB, Connoly MT. Progress in elder abuse screening and assessment instruments. J Am Geriatr Soc 2004; 52:297-304.

21. Nelson HD, Nygren P, McInerney Y, Klein J. Screening women and elderly adults for family and intimate partner violence: a review of the evidence for the U.S. preventive services task force. Ann Intern Med 2004; 140:387-96.

22. Johnson TF. Elder mistreatment: deciding who is at risk. Westport: Greenwood Press; 1991.

23. Johnson TJ. Identification instruments: finding common ground. In: Johnson TJ, editor. Elder mistreatment: deciding who is at risk. Westport: Greenwood Press; 1991. p. 85-115.

24. Streiner DL, Norman GR. Health measurement scales. A practical guide to their development and use. New York: Oxford Medical Publications; 1995.

25. Nunnally JC, Bernstein IH. Psychometric theory. New York: McGraw-Hill; 1994.

26. Shrout PE. Measurement reliability and agreement in psychiatry. Stat Methods Med Res 1998; 7:301-17.

27. McDowell IW, Newell C. Measuring health: a guide to rating scales and questionnaires. New York: Oxford University Press; 1996.

28. Guillemin F, Bombardier C, Beaton D. Cross-cultural adaptation of health-related quality of life measures: literature review and proposed guidelines. J Clin Epidemiol 1993; 46:1417-32.

29. Herdman M, Fox-Rushby J, Badia X. "Equivalence" and the translation and adaptation of health-related quality of life questionnaires. Qual Life Res 1997; 6:237-47.

30. Reichenheim ME, Moraes CL. Alguns pilares para a apreciação da validade de estudos epidemiológicos. Rev Bras Epidemiol 1998; 2:131-48.

31. Reichenheim ME, Moraes CL. Buscando a qualidade das informações em pesquisas epidemiológicas. In: Minayo MCS, Deslandes SF, organizadores. Caminhos do pensamento - epistemologia e métodos. Rio de Janeiro: Editora Fiocruz; 2002. p. 227-55.

32. Straus MA. Measuring intrafamiliar conflict and violence: the Conflict Tactics (CT) Scales. J Marriage Fam 1979; 41:75-88.

33. Hasselmann M, Reichenheim ME. Adaptação transcultural de versão em português da Conflict Tactics Scales Form R (CTS-1), usada pra aferir violência no casal: equivalências semânticas e de mensuração. Cad Saúde Pública 2003; 19:1083-93.

34. Rubenstein LZ. An overview of comprehensive geriatric assessment: rationale, history, program models, basic components. In: Rubenstein LZ, Wieland D, Bernabei R, editors. Geriatric assessment technology: the state of the art. Milan: Editrice Kurtis; 1995. p. 1-9.

35. Fulmer TT. Elder abuse assessment tool. Dimens Crit Care Nurs 1984; 3:216-20.

36. Fulmer TT, Gould ES. Assessing neglect. In: Baumhover LA, Beall SC, editors. Abuse, neglect 
and exploitation of older persons. Baltimore: Health Professions Press; 1996. p. 89-104.

37. Fulmer T, Paveza G, Abraham I, Fairchild S. Elder neglect assessment in the emergency department. J Emerg Nurs 2000; 216:436-43.

38. Lachs MS, Pillemer K. Current concepts: abuse and neglect of elderly persons. N Engl J Med 1995; 332:437-43.

39. Stuck AE, Siu AL, Wieland D, Adams J, Rubenstein LZ. Effects of comprehensive geriatric assessment on survival, residence and function: a metaanalysis of controlled trials. Lancet 1993; 342: 1032-6.

40. Kosberg JI, Nahmiash D. Characteristics of victims and perpetrators and milieus of abuse and neglect. In: Baumhover LA, Beall SC, editors. Abuse, neglect, and exploitation of older persons - strategies for assessment and intervention. Baltimore: Health Professions Press; 1996. p. 31-50.

41. Boult C, Pacala JT. Care of older people at risk. In: Calkins E, Boult C, Wagner EH, Pacala JT, editors. New ways to care for older people - building systems based on evidence. New York: Springer Publishing Company; 1999. p. 65-81.

42. Collins KA, Bennett AT, Hanzlick R. Elder abuse and neglect: Autopsy Committee of the College of American Pathologists. Arch Intern Med 2000; 160:1567-9.

43. Corp FM. The model of domestic elder abuse and neglect. In: Corp FM, editor. Elder abuse in the family - an interdisciplinary model for research. New York: Springer Publishing; 2000. p. 63-82.

44. Applegate WB, Blass JP, Williams TF. Instruments for the functional assessment of older patiens. N Engl J Med 1990; 322:1207-14.

45. American Medical Association. Diagnostic and treatment guideline on elder abuse and neglect. Chicago: American Medical Association; 1992.

46. Bass DM, Anetzberger GJ, Ejaz FK, Nagpaul K. Screening tools and referral protocol for stopping abuse against older Ohioans: a guide for service providers. Journal of Elder Abuse \& Neglect 2001; 13:23-8.

47. Lachs MS. Preaching to the unconverted: educating physicians about elder abuse. Journal of Elder Abuse \& Neglect 1995; 4:1-12.

48. Rosenblatt DE. Documentation. In: Baumhover LA, Beall SC, editors. Abuse, neglect and exploitation of older persons; strategies for assessment and intervention. Baltimore: Health Professions Press; 1996. p. 145-62.

49. Fulmer TT, O'Malley TA. The difficulty of defining abuse and neglect. In: Fulmer TT, O'Malley TA, editors. Inadequate care of the elderly. New York: Springer Publishing; 1987. p. 13-24.

50. Schofield MJ, Reynolds R, Mishra GD, Powers JR, Dobson AJ. Screening for vulnerability to abuse among older women: women's health Australia study. J Appl Gerontol 2002; 21:24-39.
51. Meeks-Sjostrom D. A comparison of three measures of elder abuse. J Nurs Scholarsh 2004; 36:24750.

52. Reis M, Nahmiash D. When seniors are abused: an intervention model. Gerontologist 1995; 35: 666-71.

53. Reis M, Nahmiash D. Validation of the Caregiver Abuse Screen (CASE). Can J Aging 1995; 14:45-60.

54. Neale AV, Hwalek MA, Scott RO, Sengstock MC, Stahl C. Validation of the Hwalek-Sengstock Elder Abuse Screening Test. J Appl Gerontol 1991; 10:406-18.

55. Hwalek MA, Sengstock MC. Assessing the probability of abuse of the elderly: toward development of a clinical screening instrument. J Appl Gerontol 1986; 5:153-73.

56. Reis M, Nahmiash D. Validation of the Indicators of Abuse (IOA) screen. Gerontologist 1998; 38:47180.

57. Phillips LR, Morrison EF, Chae YM. The QUALCARE Scale: developing an instrument to measure quality of home care. Int J Nurs Stud 1990; 27:61-75.

58. Phillips LR, Morrison EF, Chae YM. The QUALCARE Scale: testing of a measurement instrument for clinical practice. Int J Nurs Stud 1990; 27:77-91.

59. McFarlane J, Parker J, Soeken K, Bullock L. Assessing for abuse during pregnancy. JAMA 1992; 267:3176-8.

60. Herdman M, Fox-Rushby J, Badia X. A model of equivalence in the cultural adaptation of HRQol instruments: the universalist approach. Qual Life Res 1998; 7:323-35.

61. Jones J, Dougherty J, Schelble D, Cunningham W, Akron. Emergency department protocol for the diagnosis and evaluation of geriatric abuse. Ann Emerg Med 1988; 17:1006-15.

62. Reis M, Nahmiash D. Abuse of seniors: personality, stress and other indicators. J Ment Health Aging 1997; 3:337-56.

63. Davies M. Key issues for nursing: the need to challenge practice. In: Decalmer P, Glendenning F, editors. The mistreatment of elderly people. London: Sage Publications; 1997. p. 175-85.

64. Davies M. Recognizing abuse: an assessment tool for nurses. In: Decalmer P, Glendenning F, editors. The mistreatment of elderly people. London: Sage Publications; 1997. p. 102-6.

65. Fulmer T, Gurland B. Restriction as elder mistreatment: differences between caregiver and elder perceptions. J Ment Health Aging 1996; 2:89-100.

66. Ferguson D, Beck C. H.A.L.F.: a tool to assess elder abuse within the family. Geriatr Nurs 1983; 4:301-4.

67. Hamilton GP. Preventing elder abuse: using a family systems approach. J Gerontol Nurs 1989; 15:21-6.

Recebido em 21/Jul/2005

Versão final reapresentada em 03/Nov/2005

Aprovado em 07/Nov/2005 